\title{
Décomposition de la croissance en hauteur du pin maritime (Pinus pinaster Ait.)

\author{
Variabilité géographique \\ des composantes morphogénétiques et phénologiques
}

\author{
A. KREMER et G. ROUSSEL \\ I.N.R.A., Laboratoire d'Amélioration des Arbres forestiers \\ Pierroton, F 33610 Cestas
}

\section{Résumé}

L'accroissement annuel en hauteur chez le pin maritime est décomposé en quatre étapes successives de la pousse jusqu'au niveau du méristème apical. L'objectif est de rechercher les composantes permettant d'expliquer à chaque niveau de décomposition la variation du caractère "résultante ». Cette décomposition est appliquéc à neuf provenances originaires de l'ensemble de l'aire naturelle.

Au vu des valeurs des coefficients de corrélation entre composantes et caractère "résultante», la pousse primaire, correspondant au premier cycle morphogénétique, est un meilleur prédicteur de la longueur de la pousse annuelle que la pousse secondaire; le nombre d'entre-nœuds est un meilleur prédicteur de la longueur de la pousse primaire que la longueur moyenne d'un entre-noud.

Des différences importantes entre provenances sont trouvées pour la durée et l'intensité de fonctionnement des méristèmes responsables de l'initiation et de l'élongation des entrenœuds. Enfin, les taux de croissance dans le sens radial des primordia et du dôme apical au niveau du méristème sont bien corrélés avec le nombre total d'entre-nouds formés une année donnée.

Mots clés : Décomposition de la croissance en hauteur, morphogenèse, phyllotaxie, polycyclisme, durée de plastochron, provenance, pin maritime.

\section{Introduction}

Chez les conifères, l'accroissement en hauteur se décompose successivement en plusieurs unités morphologiques : cycle, entre-næud, cellule. La part prise par chacune d'entre elles dans l'accroissement final dépend de leur nombre et de leur longueur respective. Le nombre d'unités résulte de la durée et de l'intensité de leur initiation, la longueur des unités résulte de la durée et de l'intensité de leur élongation. Ainsi dans le cas du pin maritime, l'accroissement annuel en hauteur peut être subdivisé en composantes morphogénétiques ou phénologiques. Cette décomposition 
se place à quatre niveaux successifs de la pousse annuelle à la cellule (fig. 1). On appellera dans le texte caractère "résultante» le caractère somme ou produit de plusieurs composantes.

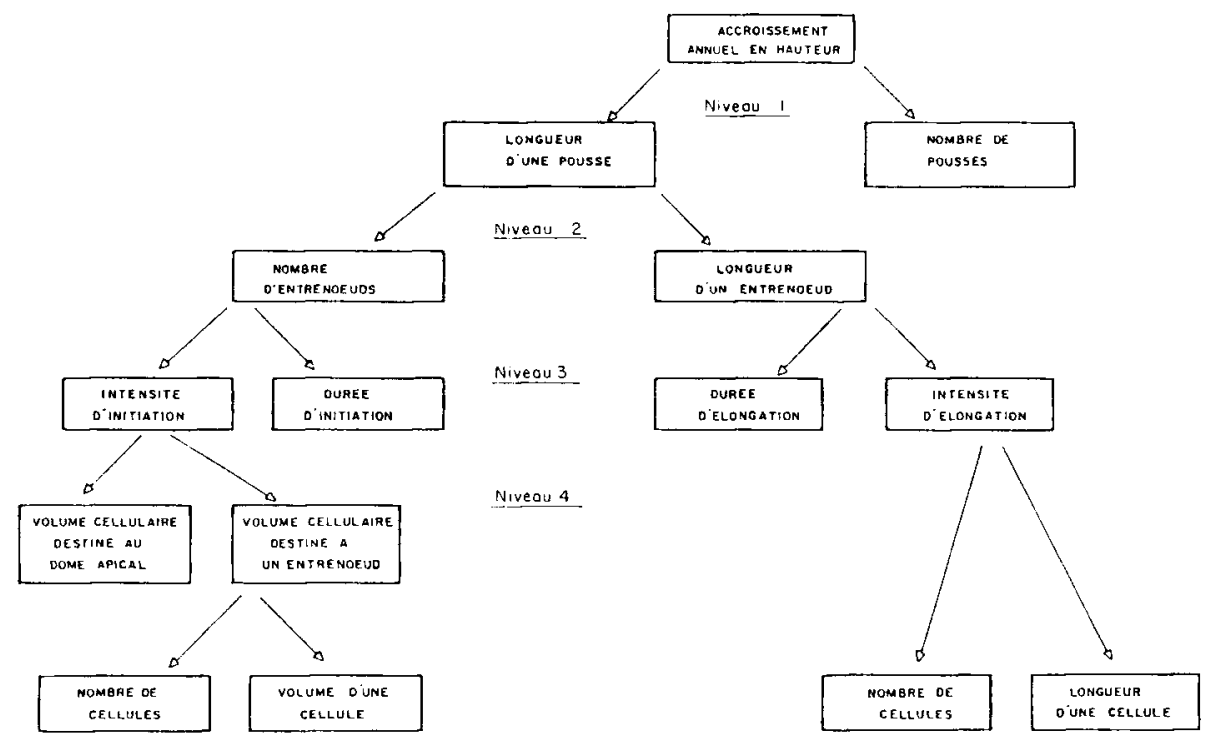

FIG. 1

Décomposition de l'accroissement annuel en hauteur (d'après CanNell, 1978).

Subdivision of the annual shoot increment (from CANNELL, 1978).

Ce schéma de décomposition est basé sur des observations et des résultats expérimentaux qui sont brièvement résumés. Un cycle morphogénétique correspond à une succession d'entre-næuds portant à leur extrémité des écailles dont les structures axillaires suivent une séquence donnée (absence de structures, brachyblastes, cônes, auxiblastes) (Debazac, 1963). Le nombre de cycles morphogénétiques présents sur une pousse annuelle est de deux ou de trois (KrEmER, 1982). En dehors de la période juvénile, les entre-nœuds s’allongeant une année donnéc sont tous initiés dans le bourgeon formé l'année précédente. L'initiation d'un nouveau primordium d'écaille associé à un entre-nœud s'accompagne de la formation par le méristème apical de cellules, destinćes d'une part au nouveau primordium et d'autre part au dôme apical, partie du méristème dépourvue de primordia (Kremer, 1984 (a) ; Cannell, 1976; Gregory Romberger, 1972). En effet ces travaux montrent que le dôme apical accroît son propre volume pendant la saison de végétation. Cette observation impose un niveau supplémentaire de décomposition entre l'entre-nœud et les cellules qui sépare le volume cellulaire destiné à un nouveau primordium de celui destiné au dôme apical.

L'intérêt de la décomposition d'un caractère complexe tel que la croissance en hauteur est triple:

- au niveau des connaissances, par l'étude des relations entre composantes, elle permet de mieux comprendre le développement et la morphogenèse de l'arbre ; 
- au niveau de la sélection et de l'amélioration génétique d'une espèce elle peut être une voie de recherche de critères prédicteurs de la croissance ;

- au niveau de l'élaboration d'une stratégie de recherche, elle permet d'identifier des facteurs clés, ou composantes, contribuant pour une majeure part à un caractère économique intéressant.

La présente étude se propose de réaliser la décomposition de laccroissement annuel en hauteur jusqu'au niveau du fonctionnement du méristème apical chez neuf provenances de pin maritime issues de l'ensemble de l'aire naturelle. Les résultats obtenus sont interprétés en fonction des trois objectifs précédemment affichés.

\section{Matériel et méthodes}

\subsection{Matériel végétal}

L'étude porte sur neuf provenances de l'ensemble de l'aire naturelle (fig. 2). Elles peuvent se regrouper en cinq groupes selon leur origine, à savoir : provenances atlantiques, corses, méditerranéennes, ibériques et marocaine. Ce matériel a été installé à l'automne 1970 en lande mésophile sur le domaine de l'Hermitage (Pierroton) dans un test de provenances comprenant au total 16 provenances (GuYoN, 1980). Le dispositif est une lattice $4 \times 4$ répété cinq fois et lui même répété 4 fois; il comprend donc au total 20 répétitions (13 plants par provenance et par répétition). L'échantillonnage de 9 provenances est basé sur une étude précédente (GuYon, 1980 ; GuYoN \& KrEmEr, 1982) montrant que celles-ci étaient représentatives des cinq groupes cités pour la croissance en hauteur.

\subsection{Mesures}

\subsection{Décomposition ant niveau I}

Les accroissements annuels en hauteur ont été mesurés de la 4 " à la $9^{\text {" saison }}$ de végétation sur 40 arbres par provenance, échantillonnés systématiquement à raison de 2 arbres par provenance et par répétition. La présence d'un pseudo-verticille d'auxiblastes permet d'identifier un cycle morphogénétique (DEbazac, 1963: Kremer \& RousSEL, 1982).

En utilisant ce marqueur morphologique, on a décomposé chaque accroissement annuel en accroissement primaire et secondaire, correspondant respectivement au premier et au second cycle morphogénétique. Cette décomposition permet ainsi de dénombrer les seconds cycles sur l'ensemble des six années prises en compte.

\subsection{Décomposition au niveau 2}

D'avril à septembre 1980, à l'occasion d'une éclaircie systématique dans le test de provenances, des prélèvements destructifs ont été effectués sur les arbres éliminés et appartenant aux provenances étudiées $(60$ à 70 arbres par provenance soit un échantillonnage systématique de trois arbres par provenance et par répétition). Les écailles ont été dénombrées sur le premier cycle morphogénétique en élongation. Ce nombre correspond également au nombre d'entre-nœuds. 


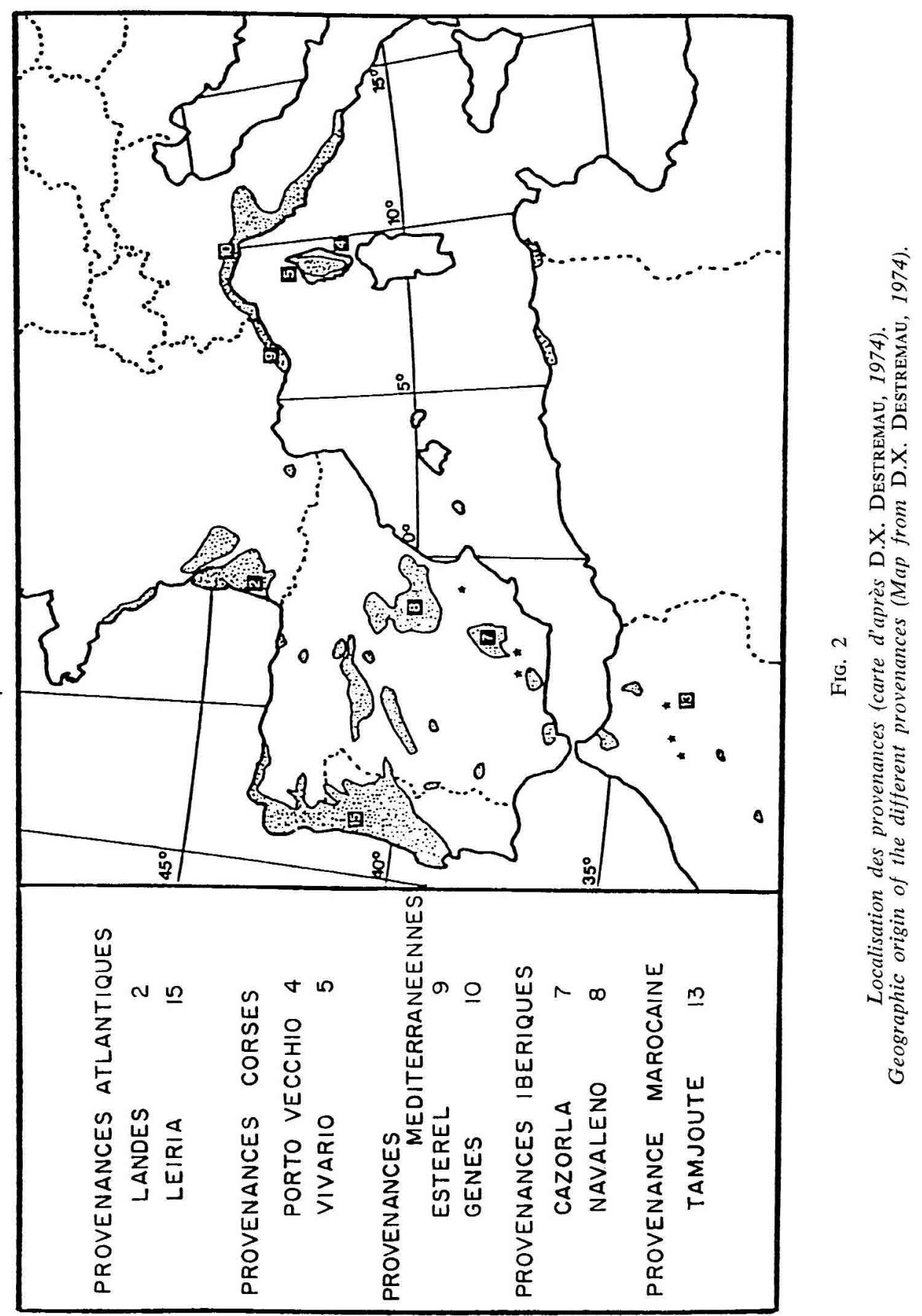




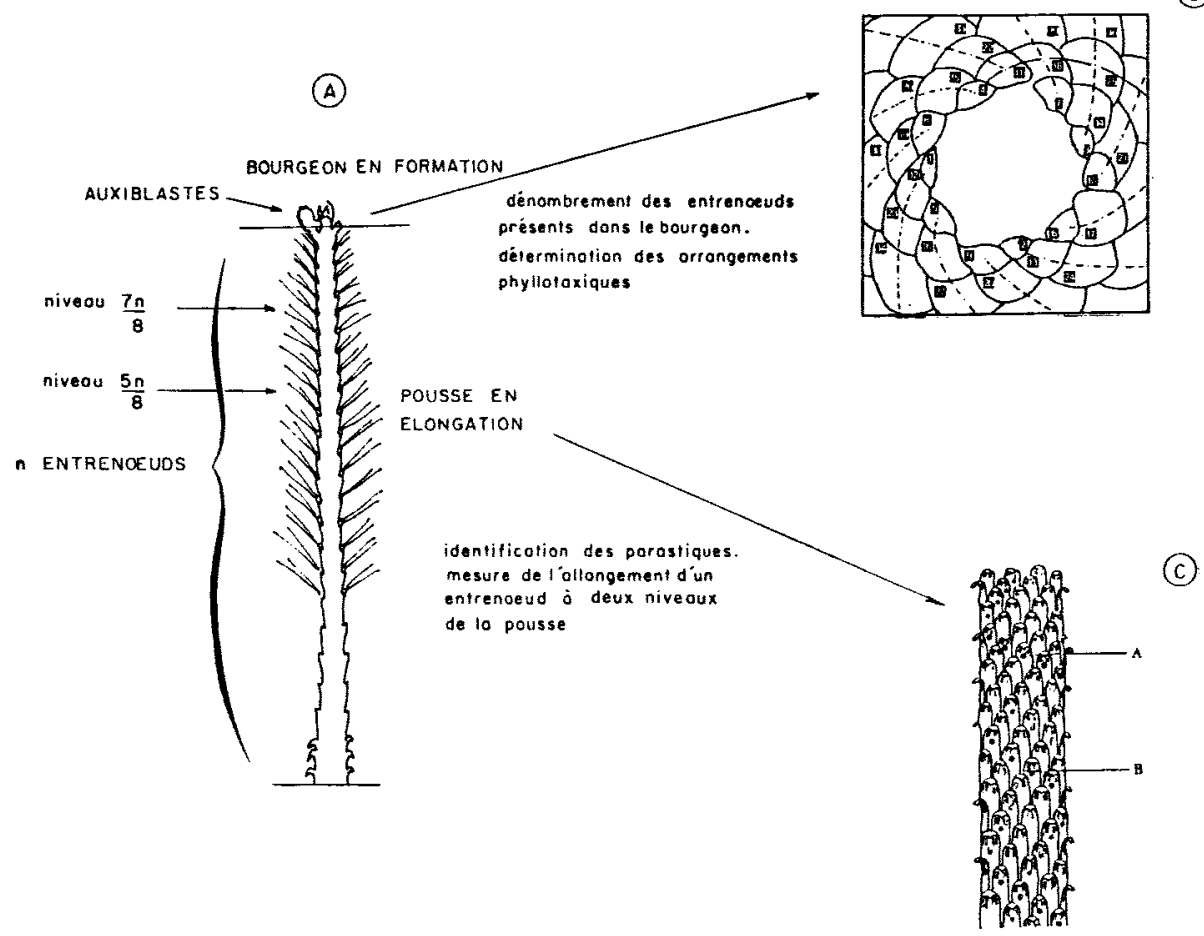

(B)

Fig. 3

Représentation schématique des mesures effectuées.

Diagram of the measurements taken.

A - Description morphologique des parties de l'appareil végétatif prélevées. Le niveau des mesures de longueur d'entre-næud est indiqué.

B - Vue schématique d'une projection horizontale du méristème apical. Les primordia d'écailles sont dénombrés par comptage direct lors de la dissection. Les 20 derniers primordia sont laissés intacts pour les mesures des taux de croissance dans le méristème (voir figure 4).

C - Grossissement de la partie de la tige utilisée pour les mesures de la longueur d'un entre-noud. Les points $A$ et $B$ sont situés sur un même parastique de contact ( 5 dans ce cas). $\mathrm{Si} x$ est le nombre d'écailles séparant ces deux points sur ce parastique, $\mathrm{AB} / 5 \mathrm{x}$ est la moyenne de la longueur d'un entre-nœud à ce niveau.

A - Morphological description of shoot sections harvested for measurements. Level of mean stem unit length measurement is indicated.

$B$ - Diagram of a transverse projection of an apical meristem. Cataphyll primordia are counted during dissection. The twenty last formed cataphylls are not removed in order to use them for measurements of radial relative growth rates.

$C$ - Magnification of the part of the shoot used for mean stem unit length measurement. Points $A$ and $B$ are located on the same contact parastichy $(5$ in this example) If $x$ is the number of scales separating the two points then $A B / 5 x$ is the measure of the mean stem unit length at this level. 
La longueur d'un entre-næud est obtenue en faisant le rapport de l'ćlongation totale sur le nombre d'entre-nouds.

\subsection{Décomposition at nivealt 3}

Des prélèvements destructifs ont été faits à 13 reprises espacées de une ou de deux semaines entre le 21 avril et le 29 septembre. A chaque récolte, 5 répétitions ont été tirćes au sort et un arbre par répétition et par provenance a été retenu pour l'étude (5 arbres par provenance et par récolte).

Sur chaque arbre abattu, a été prélevé l’ensemble formé par la pousse annuelle en élongation correspondant à la $11^{\circ}$ saison de végétation et la pousse embryonnaire en formation correspondant à la 12" saison de végétation. Dès la récolte, cet ensemble a été séparé en deux parties (fig. 3).

- Sur la pousse en ćlongation, la longueur des entre-nœuds a été mesurée à quatre niveaux différents sur la pousse primaire en utilisant les arrangements phyllotaxiques.

Si n est le nombre total d'entre-nouds se trouvant sur le premier cycle, la longueur d'un entre-nœud a été mesuré aux $\underset{3}{\mathrm{n}}$ deux $\underset{\mathrm{n}}{\text { niveaux }}$ suivants $: \frac{5 \mathrm{n}}{8}$ et $\frac{7 \mathrm{n}}{8}$. Les mesures faites au niveau inférieur $\left(\frac{3 n}{8}\right.$ et $\left.\frac{n}{8}\right)$ n'ont pu être exploitées : l'élongation des entre-nœuds correspondants était à un stade trop avancé.

- Sur la pousse en formation, après fixation au FAA, le bourgeon a été disséqué et les écailles ont été dénombrées, jusqu'au niveau du dôme apical. Ce nombre correspond aussi au nombre d’entre-nœuds présents dans le bourgeon. L'entre-nœud est pris ici au sens anatomique et correspond à la portion de tige située entre deux écailles successives et non pas entre deux pseudo-verticilles.

Les mesures répétées dans le temps permettent ainsi de construire les courbes de l'évolution de l'initiation et de l'élongation. Les moyennes par provenance des données recueillies à chaque récolte ont été ajustées à la fonction logistique généralisée de Richards (Ricitards, 1959 ; Causton \& Venus, 1981 ; Lebreton \& Millier, 1982). Cette fonction permet de déterminer trois paramètres : la durée de fonctionnement des méristèmes, l'intensité de fonctionnement et le temps où l'intensité maximale de fonctionnement est acquise (point d'inflexion de la courbe).

\subsection{Décomposition atu niveau 4}

I.es mesures concernent les estimations du volume cellulaire destiné à l'initiation d'un entre-nœud et de celui destiné à l'accroissement du dôme apical lui-même. Les principaux travaux faits sur le fonctionnement du méristème apical chez les conifères ont montré que les variations de volume du méristème étaient principalement liées à celles du rayon dans le plan transversal (fig. 4), (Gregory \& Romberger, 1972; Kremer, 1984 a). Sur la projection dans le plan transversal, les primordia sont disposés sur une spirale logarithmique (Williams, 1975; JEAN, 1978; Thornley, 1975) caractérisée par la relation suivante :

$$
\mathbf{R}_{\mathrm{i}}=\mathbf{R}_{0} \mathrm{k}^{\mathrm{i}}
$$

$R_{i}=$ rayon du primordium $i$ par rapport au centre de la spirale.

$\mathbf{R}_{\text {, }}=$ rayon du dôme apical par rapport au centre de la spirale.

$\mathrm{k}=$ constante. 


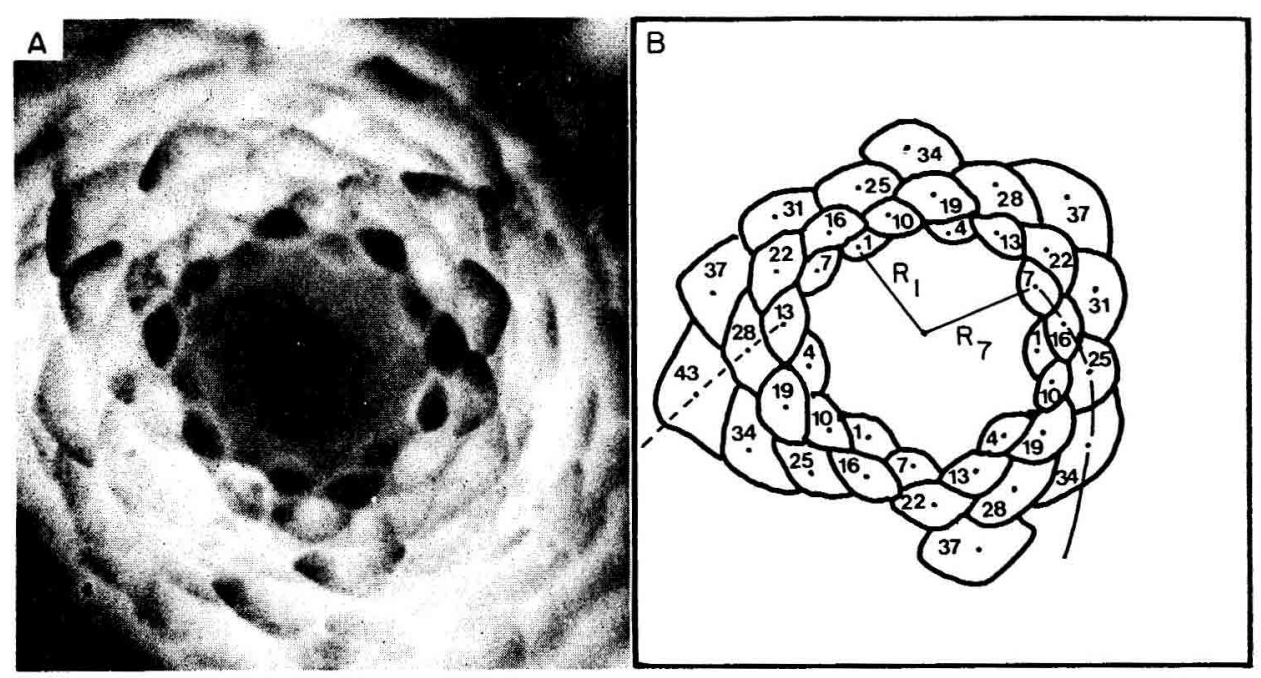

FIG. 4

Illustration du principe d'identification des arrangements phyllotaxiques.

Example showing the principles used for identification of phyllotactic arrangements.

A - Photographie d'une vue horizontale d'un méristème apical de pin maritime $(\times 75)$ prise sous la loupe binoculaire. Le méristème est laissé dans le F.A.A. pour éviter des retraits dus au dessèchement. Une technique d'hypo illumination à l'aide de fibre optique est adaptée à la loupe pour éviter les reflets.

B - Graphique réalisé à la chambre claire à partir de la photographic précédente. 9 parastiques dextres et 15 parastiques senestres peuvent aisément être identifiés. Un parastique est une courbe fictive joignant des primordia ayant des contacts de même nature. En notant 1 le plus petit primordium, le suivant situé sur le même parastique dextre sera 10 et le suivant situé sur le même parastique senestre sera 16. A partir de cet arrangement on peut ainsi numéroter tous les primordia dans leur séquence ontogénique. On peut noter que la phyllotaxie de ce méristème est multijuguée.

A - Photomicrograph of a horizontal projection of a maritime pine apical meristem ( $\times 75)$, taken on a stereo microscope. To avoid cell shrinkages, meristems are kept in F.A.A. during observation. A particular device for "hypoillumination» using an optic fiber is adapted to the stereomicroscope in order to avoid light reflexion.

$B$ - Diagram drawn with a camera lucida from the previous photomicrograph. 9 right and 15 left contact parastichies can be identified. A contact parastichy is a fictive curve joining primordia of similar contacts. If 1 is the most recent initiated primordium, then the next one located on the same right contact parastichy is 10 while the next one on the same left contact parastichy is 16. This principle is then used to number all the primordia in their ontogenic sequence. Note that the meristem exhibits a multijugate phyllotaxy.

Dans le plan transversal, on définit le primordium i correspondant à l'entre-nœud i comme la couronne de largeur $R_{i+1}-R_{i}$ et le dôme apical comme le cercle de rayon $\mathrm{R}_{0}$.

$$
R_{i+1}-R_{i}=R_{o}(k-1) k^{i}
$$

Une évaluation indirecte des volumes cellulaires destinés à l'initiation d'un primordium et à l'accroissement du dôme apical est donnée par les taux relatifs de crois- 
sance dans le sens transversal des primordia $\left(r_{p}\right)$ et du dôme $\left(r_{d}\right)$ par darée de plastochron $(\mathrm{T})$, temps séparant l'initiation de deux primordia successifs.

$$
\begin{gathered}
r_{n}=\frac{d R_{o}}{R_{0} d T}+\frac{d k}{(k-1) d T}+\frac{d^{i}}{k^{i} d T} \\
r_{d}=\frac{d R_{0}}{R_{0} d T}
\end{gathered}
$$

En ce qui concerne $r_{p}$, les résultats expérimentaux montrent que les deux premiers termes de sa définition $\frac{\mathrm{dR}_{0}}{\mathrm{dR}_{0} \mathrm{dT}}$ et $\frac{\mathrm{dk}}{(\mathrm{k}-1) \mathrm{dT}}$ sont de signe différent, de valeur absolue proche et très inférieure à celle du troisième terme (KrEmER, 1984 a). On peut donc écrire :

$$
r_{10} \simeq \frac{d^{i}}{k^{i} d T}
$$

Par définition on peut écrire $\mathrm{di}=\mathrm{dT}$

$$
\mathrm{r}_{\mathrm{p}} \simeq \log \mathrm{k}
$$

$\mathrm{k}$, qui est une constante de la spirale logarithmique, peut donc être mesurée à partir d'une observation ponctuelle des arrangements phyllotaxiques. posée.

En ce qui concerne $r_{4}$ une mesure moyenne sur une période d'activité est pro-

Si $R_{\text {mnin }}$ est le rayon du dôme apical quand l'activité du méristème apical est minimale (début de saison de végétation), $R_{\text {malix }}$ le rayon quand l'intensité d'initiation est maximale et $\mathrm{N}$ le nombre d'entre-nœuds initiés durant cette période, une mesure moyenne de $r_{i l}$ sur cette période est donnée par $r^{\prime}$.

$$
r_{1}^{\prime}=\frac{R_{\text {11nax }}-R_{\text {omin }}}{R_{\text {omin }} N}
$$

Les deux estimations de $r_{11}$ et de $r_{1}^{\prime}$ ne se placent donc pas au même niveau. $r_{p}$ est une mesure ponctuelle à l'ćchelle de la durée d'un plastochron, alors que $r^{\prime}{ }_{a}$ est une moyenne sur une période d'initiation plus longue.

Les estimations de $r_{1}$ et de $r^{\prime}$, nécessitent donc les mesures de $\mathbf{R}_{0}$ et de $\mathbf{k}$ (équation 1). Elles sont basées sur l'interprétation géométrique des arrangements phyllotaxiques; cette analyse est détaillée dans une étude précédente (KREMER, 1984 a). On ne rappelle ici que les principales étapes:

- récolte des bourgeons sur 5 arbres par provenance à 13 reprises entre le 21 avril et le 29 septembre ;

- dissection de chaque bourgeon jusqu'au niveau du dôme apical en ne laissant que les 20 derniers primordia; 
- représentation graphique à l'aide de la chambre claire de la projection transversale du méristème (fig. $4 \mathrm{~B}$ ) ;

- identification des parastiques de contact : 9 dans le sens dextre, 15 dans le sens senestre dans le cas du méristème représenté sur la figure $4 \mathrm{~B}$. Un parastique ou parastique de contact est une courbe fictive joignant des primordia ayant des contacts de même nature ;

- numérotation de tous les primordia dans leur séquence ontogénique en utilisant les parastiques de contact ;

- relevé des coordonnées de chaque centre de primordia pris à la table à numériser ;

- mesure des $R_{i}$, à partir d'un programme informatique utilisant les données fournies par la table à numériser ;

- régression linéaire de $\log R_{i}$ sur $i$ qui permet l'estimation de $R_{0}$ et de $k$.

\section{Résultats}

\subsection{Décomposition de la pousse annuelle (niveau 1)}

Les moyennes des accroissements totaux, primaires, secondaires, et le nombre des pousses secondaires sur 6 années succesives sont données dans le tableau 1 pour chaque provenance.

\section{Tableau 1}

Moyennes des sommes des accroissements totaux, primaires et secondaires chez différentes provenances de pin maritime.

Provenance mean values of the sum of total,

primary and secondary shoot increments of different provenances of maritime pine.

\begin{tabular}{|c|c|c|c|c|}
\hline Provenances & $\begin{array}{c}\text { Accroissements } \\
\text { totaux (a) }\end{array}$ & $\begin{array}{c}\text { Accroissements } \\
\text { primaires (:i) }\end{array}$ & $\begin{array}{l}\text { Accroissements } \\
\text { secondaires (a) }\end{array}$ & $\begin{array}{c}\text { Nombre } \\
\text { des pousses } \\
\text { secondaires (b) }\end{array}$ \\
\hline Landes ........... & 520 & 421 & 99 & 3,98 \\
\hline Leiria $\ldots \ldots \ldots \ldots$ & 509 & 430 & 79 & 2,78 \\
\hline Porto-Vecchio . . . . . & 455 & 390 & 66 & 2,82 \\
\hline Vivario $\ldots \ldots \ldots \ldots$ & 441 & 376 & 65 & 2,87 \\
\hline Esterel .......... & 437 & 372 & 65 & 2,92 \\
\hline Gênes $\ldots \ldots \ldots \ldots$. & 408 & 342 & 67 & 3,78 \\
\hline Cazorla $\ldots \ldots \ldots$ & 414 & 379 & 34 & 1,50 \\
\hline Navaleno ......... & 423 & 384 & 39 & 1,93 \\
\hline Tamjoute $\ldots \ldots \ldots$ & 371 & 320 & 51 & 2,59 \\
\hline$\ldots \ldots \ldots$ & $44,4 * *(0)$ & $31,3 *(x)$ & $7,4 * *(c)$ & $8,7 *(\cdot)$ \\
\hline
\end{tabular}

(a) Accroissements cumulés sur 6 années successives (4ª̀ la $9^{\text {c }}$ saison de végćtation) exprimés en centimètres - Shoot increments summed over 6 successives years, from the 4th to the 9th growing season. Shoot increments are given in centimeters.

(b) Nombre de seconds cycles exprimés en 6 ans - Number of secondary shoots expressed during the 6 years.

(c) *:* Test $F$ significatif au seuil de 1 p. 100. 
Ces résultats montrent qu'il n’existe pas de liaison entre l'origine des provenances et l'expression d'un second cycle de croissance. Parmi les provenances les plus polycycliques, on trouve une provenance landaise et une provenance italienne. Par contre, les provenances les moins polycycliques sont issues de la partie centrale de la péninsule ibérique. On note enfin que la provenance marocaine a une fréquence d'expression de seconds cycles aussi élevée que la provenance portugaise Leiria.

Le second résultat intéressant correspondant à ce niveau de décomposition est la corrélation élevée entre accroissement primaire et accroissement total (tabl. 2).

\section{TABLEAU 2}

Matrice des coefficients de corrélation entre moyennes de provenancess des différents accroissements.

Correlation coefficient matrix between provenance mean values of different shoot increments.

\begin{tabular}{|c|c|c|c|}
\hline & $\begin{array}{l}\text { Accroissements } \\
\text { totaux }\end{array}$ & $\begin{array}{l}\text { Accroissements } \\
\text { primaires }\end{array}$ & $\begin{array}{l}\text { Accroissements } \\
\text { secondaires }\end{array}$ \\
\hline Accroissements primaires .... & $0,97 *$ & & \\
\hline Accroissements secondaires .. & $0,75 \%$ & 0,57 & \\
\hline $\begin{array}{r}\text { Nombre de poussses secon- } \\
\text { daires } \ldots \ldots \ldots \ldots \ldots \ldots \ldots\end{array}$ & 0,39 & 0,17 & $0,85^{*}:$ \\
\hline $\begin{array}{l}\because \text { Test significatif au seuil } \\
* \quad \text { Test significatif au seuil }\end{array}$ & $\begin{array}{l}5 \text { p. } 100 \\
1 \text { p. } 100\end{array}$ & & \\
\hline
\end{tabular}

\subsection{Décomposition de la pousse primaire (niveau 2)}

L'accroissement moyen correspondant à la pousse primaire a été obtenu en prenant la moyenne sur les 6 années prises en compte. Par contre, les entre-nouds n'ont été dénombrés que lors d'une seule saison. Le rapport entre ces deux moyennes ne correspond donc pas à la longueur d'un entre-nœud une année donnée. Mais dans la mesure où le classement des provenances pour les deux premiers caractères est stable d'une année à l'autre (GuYon \& KREMER, 1982), on peut accorder un certain crédit aux valeurs de ce rapport pour l'étude des différences entre provenances (tabl. 3).

Ce tableau montre que la variabilité entre provenances est plus importante pour le nombre d'entre-nœuds que pour les caractères d'accroissement, que ce soit l'accroissement primaire proprement dit ou la longueur moyenne d'un entre-nœud.

Deux résultats apparaissent très nettement sur le tableau 4 qui représente les coefficients de corrélation entre composantes de l'accroissement primaire : $(0,92)$;

- la corrélation élevée entre le nombre d'entre-nœuds et l'accroissement primaire

- la liaison négative significative entre les deux composantes. 


\section{TABleau 3}

Moyennes des accroissements primaires, du nombre d'entre-nouds et de l'élongation moyenne d'un entre-noud chez différentes provenances de pin maritime.

Provenance mean values of primary shoot increments,

Number of stem units and mean stem unit length of different maritime pine provenances.

\begin{tabular}{|c|c|c|c|}
\hline Provenances & $\begin{array}{c}\text { Accroissement } \\
\text { primaire annuel (a) }\end{array}$ & $\begin{array}{c}\text { Nombre } \\
\text { d'entre-nœuds (b) }\end{array}$ & $\begin{array}{l}\text { Elongation moyenne } \\
\text { d'un entre-nœud (c) }\end{array}$ \\
\hline Landes & 70,1 & 448 & 1,56 \\
\hline Leiria $\ldots \ldots \ldots \ldots$ & 71,6 & 527 & 1,36 \\
\hline Porto Vecchio . .... & 64,9 & 428 & 1,52 \\
\hline Vivario $\ldots \ldots \ldots$ & 62,7 & 415 & 1,51 \\
\hline Esterel & 61,9 & 395 & 1,57 \\
\hline Gênes ....... & 57,0 & 390 & 1,46 \\
\hline Cazorla .. & 63,2 & 407 & 1,55 \\
\hline Navaleno .. & 64,0 & 423 & 1,52 \\
\hline Tamjoute ........ & 53,4 & 272 & 1,96 \\
\hline rest $\mathrm{F}$ & $30,4^{*} *(\boldsymbol{d})$ & $35,1 * *(d)$ & Non calculé \\
\hline
\end{tabular}

(a) Moyenne des accroissements primaires sur 6 ans (4" à $9^{\mathrm{r}}$ saison de végétation) exprimée en centimètres - Mean values of primary shoot increments over 6 successive years from the 4 th to the 9th growing season, expressed in centimeters.

(b) Dénombré lors de la onzième saison de végétation - Number of stem units counted auring the eleventh growing season.

(c) Rapport entre les deux précédentes mesures, exprimé en millimètres. - Ratio of the two previous measurements, expressed in millimeters.

(d) *:* Test F significatif au seuil de 1 p. 100.

\section{Tableau 4}

Matrice des coefficients de corrélation entre moyennes de provenance de l'accroissement primaire,

du nombre d'entre-nouds et de l'élongation moyenne d'un cntre-noud.

Correlation coefficient matrix between provenance mean values of primary shoot increments, number of stem units and mean stem unit length.

\begin{tabular}{|c|c|c|}
\hline & $\begin{array}{l}\text { Accroissement } \\
\text { primaire }\end{array}$ & Nombre d'entre-nœuds \\
\hline Nombre d'entre-nœuds & $0,92^{\text {*** }}$ & \\
\hline Elongation d'un entre-nœud & $-0,68^{*}$ & $-0,89 * *$ \\
\hline
\end{tabular}

* Test significatif au seuil de 5 p. 100.

** Test significatif au seuil de 1 p. 100. 
Ainsi, la provenance Tamjoute dont la pousse primaire ne comporte qu'un nombre faible d'entre-nœuds est celle pour laquelle ces entre-nœuds sont les plus longs.

\subsection{Décomposition phénologique de l'initiation et de l'élongation (niveau 3)}

Pour la décomposition phénologique, les provenances ont été regroupées par deux comme il est indiqué sur la figure 2. On dispose donc de 10 observations par récolte et pour chaque groupe de provenances.

La méthode d'ajustement utilisée est celle des moindres carrés non linéaires (CAuston \& Venus, 1981). La méthode consiste, après recherche des valeurs de départ des 4 paramètres de la fonction, à procéder par itération jusqu'à ce que la somme des carrés des écarts soit minimale. Il n'a pas été effectué de pondération compte tenu de l'hétéroscédasticité des données.

Les rythmes d'initiation et d'élongation des entre-nouds sont représentés sur la figure 5. Il s'agit de la dérivée de la fonction de Richaros.

A ces figures s'ajoutent les donnćes du tableau 5 comprenant les valeurs de taux moyen d'initiation, de durée de fonctionnement, et la date à laquelle la fonction de RICHARDS atteint le point d'inflexion.

\section{TABLEAU 5}

Moyennes des durées et taux moyen d'initiation d'entre-nouds chez différents groupes de provenances de pin maritime.

Duration and rate of initiation of stem anits of different provenances of maritime pine.

\begin{tabular}{|c|c|c|c|c|}
\hline \multicolumn{2}{|c|}{ Provenances } & \multirow{2}{*}{$\begin{array}{c}\text { Durée } \\
\text { d'initiation } \\
178\end{array}$} & \multirow{2}{*}{$\begin{array}{c}\begin{array}{c}\text { Taux } \\
\text { d'initiation (b) }\end{array} \\
3,16\end{array}$} & \multirow{2}{*}{$\begin{array}{l}\text { Abscisse du point } \\
\text { d'inflexion de la } \\
\text { courbe d'initiation } \\
22 \text { août }\end{array}$} \\
\hline $\begin{array}{l}\text { Landes } \\
\text { Leiria }\end{array}$ & atlantiques .... & & & \\
\hline $\begin{array}{l}\text { Porto Vecchio } \\
\text { Vivario }\end{array}$ & corses $\ldots \ldots \ldots$ & 164 & 2,78 & 8 août \\
\hline $\begin{array}{l}\text { Esterel } \\
\text { Gênes }\end{array}$ & méditerranéennes & 169 & 2,74 & 8 août \\
\hline $\begin{array}{l}\text { Cazorla } \\
\text { Navaleno }\end{array}$ & ibériques ..... & 163 & 3,12 & 19 août \\
\hline Tamjoute & marocaines .... & 134 & 2,53 & 7 août \\
\hline
\end{tabular}

(a) Nombre de jours d'initiation d'cntre-nœuds durant la saison de végétation - Number of days of stem unit initiation during the growing season.

(b) Nombre moyen d'entre-nouds initiés par jour durant cette période - Mean number of stem units initiated per day during this period. 

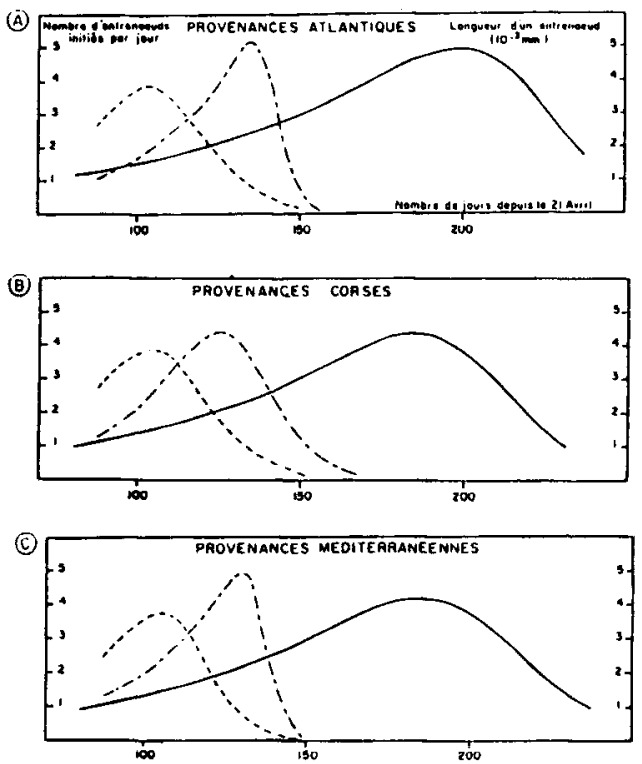

(D)
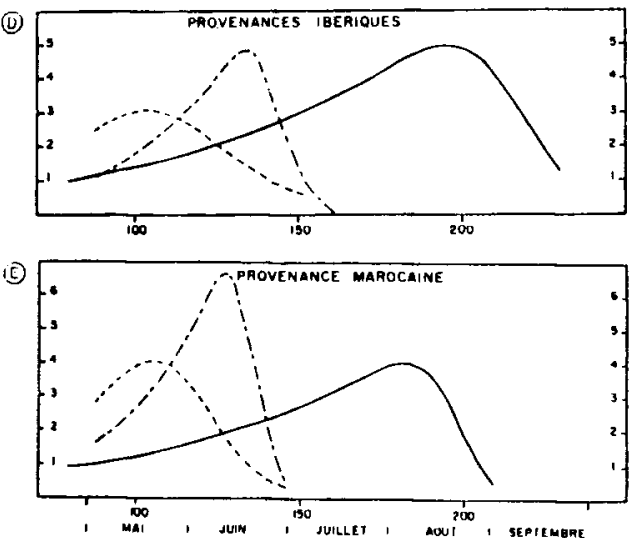

FIG. 5

Variation saisonnière du taux d'élongation et d'initiation des entre-nouds durant la onzième saison de végétation chez différentes provenances de pin maritime.

Seasonal variation of the rate of elongation and initiation of stem units in different provenances of Pinus Pinaster during the eleventh growing season.

Nombre d'entre-nœuds initiés par jour.

Number of stem units initiated per day.

Longueur de l'entre-nœud situé au niveau $\frac{5 \mathrm{n}}{8}$.

Length of the stem unit located at $\frac{5 n}{8}$ level (see figure 3).

Longueur de l'entre-noud situé au niveau $\frac{7 \mathrm{n}}{8}$.

Length of the stem unit located at $\frac{7 n}{8}$ level (see figure 3). 
Les résultats peuvent être résumés de la manière suivante :

- En ce qui concerne la phénologie du fonctionnement des méristèmes primaires, il existe un décalage net entre les populations occidentales (ibériques et atlantiques) et les populations plus orientales (corses et méditerranéennes), celles-ci étant plus précoces. Ce décalage affecte à la fois l'élongation et l'initiation des entrenœuds. La provenance marocaine se rapproche plus des dernières citées. Ces résultats apparaissent à l'observation de la localisation des points d'inflexion.

- Les durées d'initiation varient entre populations. Il y a globalement plus d'un mois de différence entre les deux extrêmes (populations atlantique et marocaine), plus d'une dizaine de jours entre les populations atlantiques et les autres. Les différences de durée sont moins évidentes en ce qui concerne l'élongation des entre-nouds.

D’une manière générale, il apparaît que les différences d'intensité d'initiation sont plus importantes que les différences de durées d'initiation.

- Chez toutes les provenances, il y a un recouvrement entre période d'élongation des entre-nouds présents dans le bourgeon et période d'initiation de ceux qui formeront le bourgeon de l'annće suivante.

- Quelles que soient les provenances, l'initiation des entre-nouds se réalise sur une longue période du mois d'avril jusquau mois de septembre et les entre-nouds sont en majorité initiés en pleine période de sécheresse estivale en juillet et août.

\subsection{Répartition des taux de crosssance dans le méristème apical}

(Décomposition au niveau 4)

Les mesures du taux relatif de croissance des primordia dans le sens radial $\left(r_{1}\right)$ ont été effectuées lors de chaque prélèvement. Les valeurs de $r_{p}$, représentées sur le tableau 6 sont des moyennes de provenances calculées à partir de mesures issues de trois prélèvements échelonnés durant la période d'initiation intense (fin juillet, début août). Ces moyennes ont donc été calculées sur la base de 15 observations (arbres) par provenance. Le choix de ces prélèvements est basé sur les courbes dintensité d'initiation de la figure 5.

En ce qui concerne $r^{\prime}{ }_{1}$ (tabl. 6), taux relatif de croissance du dôme apical dans le sens radial, les mesures de $R_{6 m i x}$ (formule 6) ont été relevées sur les arbres des trois prélèvements précédemment cités. $R_{\text {amin }}$ correspond au rayon du dôme apical mesuré lors des trois prélèvements pendant lesquels l'intensité d'initiation était minimale, au début de la saison de végétation (avril, mai sur la figure 5). Entre la période d'intensité minimale et maximale, les provenances ont en moyenne initié $70 \mathrm{p}$. 100 du nombre total de primordia formés sur l'ensemble de la période d'initiation. Ce pourcentage, estimé à partir des courbes de la figure 5 , varie peu d'une provenance à l'autre ( 85 p. 100 à 73 p. 100 selon les provenances). Cette observation permet dès lors d'estimer $N$ (équation 6 ) à partir du nombre total de primordia formés durant la période d'initiation. Les valeurs de $r^{\prime}$ sont représentées sur le tableau 6 ainsi que celles de $\mathbf{R}_{\text {omax }}$ et $\mathbf{R}_{\text {ominn. }}$.

- Les différences entre moyennes de provenance pour $r_{p}$ sont très significatives. Malheureusement, il n'a pas été possible d'obtenir des mesures au nivcau individuel (arbre) pour $r^{\prime}$ : aucun test statistique n'a dunc pu être fait pour ces valeurs. On remarque tout particulièrement que le classement des moyennes pour $r_{p}$, correspond à celui pour $r_{d}^{\prime}$. 


\section{TABLEAU 6}

Moyennes des taux relatifs de croissance et rayon du dôme apical dans les méristèmes apicaux de différentes provenances de pin maritime.

Provenance mean values of relative growth rates and radius of the bare apical dome in apical meristems of different maritime pine provenances.

\begin{tabular}{|c|c|c|c|c|}
\hline Provenance & $r_{p}^{(a)}$ & $\mathbf{r}_{\mathbf{a}}(\mathrm{b})$ & $\mathbf{R}_{\mathrm{omax}}(\mathrm{c})$ & $\mathbf{R}_{\text {omin }}$ (d) \\
\hline Landes & 13,41 & 1,18 & 424 & 309 \\
\hline Leiria $\ldots \ldots \ldots$ & 10,53 & 1,41 & 477 & 313 \\
\hline Porto Vecchio .. & 13,19 & 1,79 & 425 & 276 \\
\hline Vivario $\ldots \ldots \ldots$ & 13,87 & 1,78 & 392 & 258 \\
\hline Estercl $\ldots \ldots \ldots$ & 13,31 & 1,81 & 411 & 274 \\
\hline Gênes. & 13,51 & 2,19 & 401 & 251 \\
\hline Cazorla & 13,66 & 1,74 & 428 & 286 \\
\hline Navaleno ....... & 12,71 & 2,64 & 455 & 255 \\
\hline Tamjoute ...... & 17,13 & 2,55 & 341 & 229 \\
\hline Test F & $17,9 * *(c)$ & Non calculé & $14,2 * *$ (c) & $8,9 * *(\mathrm{c})$ \\
\hline
\end{tabular}

(a) Taux relatif de croissance des primordia dans Ie sens radial, exprimé par durée de plastochron (voir paragraphe 2.24.) donné en unité 10 - Radial relative growth rate of the primordia on a plastochron duration basis $\left(\times 10^{-3}\right)$.

(b) Taux relatif moyen de croissance du dôme apical dans le sens radial, exprimé par durée de plastochron (voir paragraphe 2.2 .4 ) donné en unité $10^{-3}$ - Mean radial relative growth rate of the bare apical dome on a plastochron duration basis $\left(\times 10^{-5}\right)$.

(c) Rayon du dôme apical en période d'initiation forte (voir paragraphe 2.2.4) donné en microns - Radius of the bare apical dome during the high initiation rale period (in micrometers).

(d) Rayon du dôme apical en période d'initiation faible (voir paragraphe 2.2.4) donné en microns - Radius of the bare apical dome during the low initiation rate period (in micrometers).

(c) $*:$ Test F significatif au seuil de 1 p. 100

\section{Tableau 7}

Matrice des coefficients de corrélation

entre moyennes des provenances des taux relatifs de croissance, rayon du dôme apical et nombre d'entre-nouds initiés.

Correlation coefficient matrix between provenance mean values of relative growth rates, radius of the bare apical dome and number of stem units.

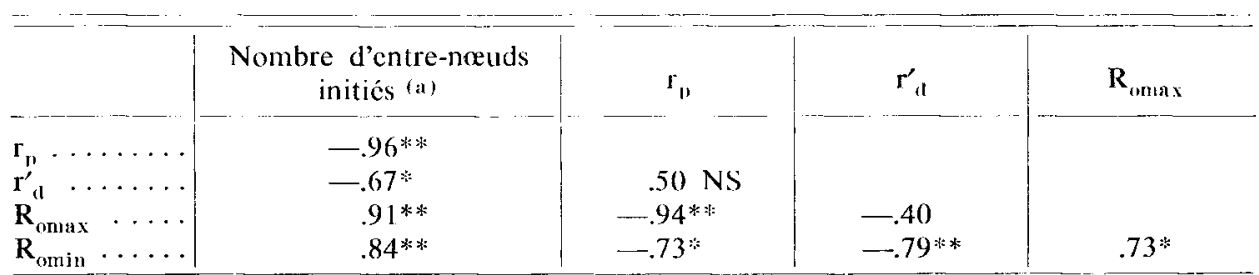

(a) Nombre d'entre-nouds allongés durant la onzième saison de végétation (voir tableau 3) -

Number of stem units elongated during the 11th growing season.

$\because$ Test significatif au seuil de 5 p. 100 .

:**: Test significatif au seuil de 1 p. 100. 
- Les différences entre moyennes de provenances de $\mathbf{R}_{\text {max }}$ sont plus importantes que celles de $R_{\text {omin }}$ à en juger par les valeurs des tests $F$. Cette remarque vaut tout particulièrement pour la comparaison entre Landes et Leiria.

En période de repos, le dôme apical de ces provenances a des dimensions voisines, alors qu'en période d'activité maximale, ces dimensions sont nettement différentes.

Les valeurs élevées de $F$ relatives au test $F$ portant sur $r_{1}$, $R_{\text {omax }}$ et $R_{\text {minin }}$ sont surtout liées aux deux extrêmes du classement à savoir les provenances Leiria et Tamjoute; les autres provenances se regroupent autour de la moyenne. Cette observation impose quelques précautions dans l'interprétation des coefficients de corrélation entre ces paramètres (tabl. 7). Néanmoins, dans ce tableau, certains coefficients de corrélation ont des valeurs particulièrement ćlevées et permettent de noter les tendances générales. Ainsi les provenances qui ont formé un nombre important d'entre-nouds se caractérisent par des dômes apicaux de grande dimension et des taux de croissance des primordia dans le sens radial faibles. La géométrie du méristème apical (rayon du dôme, arrangements phyllotaxiques) fournit ainsi une information importante sur lactivité d’initiation du méristème apical.

\section{Discussion}

La décomposition morphogénétique de l'accroissement annuel en hauteur s'avère une méthode efficace pour la recherche de caractères prédicteurs de l'élongation annuelle. En effet, quel que soit le niveau de décomposition, la variation d'une composante participe toujours avec une part plus large à celle du caractère "résultante » comme en témoignent les différents tableaux des matrices de corrélation (tabl. 2, 4 et 7). Les différentes composantes considérées à un même niveau de décomposition ne participent jamais avec une contribution égale au caractère "résultante».

Au premier niveau de décomposition, la valeur prédictrice des différentes pousses de l'accroissement annuel a rarement été étudiée dans le cas d'espèces où les deux cycles sont préformés dans le bourgeon. Pinus cont.rto correspond à cette modalité de croissance (OWens \& Molder, 1975 ; VAN DEN Berg \& Lanner, 1971): la comparaison de deux provenances l'une polycyclique, l'autre monocyclique montre que la première a une croissance annuelle plus importante (Thompson, 1974) mais léchantillonnage de provenances est bien évidemment trop faible pour conclure.

Au second niveau de décomposition, les résultats obtenus chez différentes espèces concordent. Quelle que soit l'espèce concernée (Pinus contorta, WAREING, 1970; Cannell et al., 1976; Pintes banksiana. Kremer \& Larson, 1983; Picea glatica, Pollard, 1973), et l'âge des plants, le nombre d'entre-nœuds sur une pousse est toujours mieux corrélé à la longueur de cette pousse que la longueur moyenne d'un entre-nœud. Dans ces exemples les coefficients de corrélation sont estimés à partir des moyennes de provenances. Ce résultat est d'autre part observé au niveau phénotypique à l'intérieur d'une population (KREMER, 1984 b) et au niveau famille (LAscoux, 1984, sur Pinus pinaster; Balley \& Feret, 1982, sur des hybrides de Pinus rigida $X$ Pimus taeda). 
Cette généralité permet d'ailleurs d'interpréter les relations souvent invoquées entre accroissement en hauteur une année donnée et conditions écologiques prévalant l'année précédente (KozlowsKi, 1964).

Au troisiènc niveau de décomposition, les résultats connus sont assez contradictoires. Dans deux cas, chez Picea sitchensis et Picea glauca (Canneli. \& Wirlete, 1975 ; POLlaro, 1973) le nombre total d'entre-nœuds initiés durant la saison de végétation est davantage lié à la durcé d'initation qu'au taux d'initiation alors que chez Pinus comtorta (CANNEll. \& Willet, 1975) le résultat est inverse. La nature des résultats dépend de l'extension latitudinale de l'aire naturelle ainsi que de l'échantillonnage de provenances réalisé. En effet, il y a une corrélation négative significative entre nombre d'entre-nouds et latitude d'origine de la provenance, résultat qui suggère un contrôle photopériodique de l'arrêt de l'initiation. Dans le cas d'espèce à aire naturelle morcelće et extension latitudinale réduite, les résultats peuvent être différents. Ainsi dans le cals du pin maritime, les provenances atlantiques et marocaines qui sont aux deux extrêmes du classement pour le nombre d'entre-nœuds initiés sont également aux deux extrêmes dans le même ordre pour la duréc d’initiation et le taux d'initiation (tabl. 5).

Au quatrième niveau de décomposition, nos résultats corroborent ceux obtenus dans d'autres etudes faites sur les conifères (CANNELL, 1976, sur Picea contorta; Cannili. \& Cahalan, 1979, sur Picea sitchensis; Gregory \& Rombergitr, 1972, sur Picea abies: Krlimer, 1984 a, sur Pinus banksiana). Dans tous ces exemples, il a toujours été mis en évidence une corrélation négative entre taux relatil de croissance dans le sens radial des primordia et taux dinitiation de nouveaux primordia, et $a$ fortiori nombre total d'entre-nouds initiés durant la saison de végétation. Ces résultats sont identiques à ceux observés chez le pin maritime (tabl. 7).

En résumc, et en reprenant le schéma de décomposition du niveau de la pousse jusquau niveau cellulaire, les composantes jouissant du meilleur pouvoir de prédiction du caractère «résultante» leur correspondant sont les suivants : taux de croissance des primordia dans le sens radial par durée de plastochron $\left(r_{p}\right)$, nombre d'entre-nouds et longueur de la pousse primaire. Ces paramètres sont à considérer dans toute recherche de caractères prédicteurs de la vigueur générale.

\section{Conclusion}

Parmi les résultats les plus importants, cette étude a montré que la variation du nombre d'entre-nouds prédéterminés dans le bourgeon explique la majeure partie de la variation de l'accroissement en hauteur de la pousse développée à partir de ce bourgeon. D'autre part, au vu de la figure 5, les entre-nœuds sont surtout initiés en été quand la sécheresse est la plus prononcée dans les Landes de Gascogne. On sait par ailleurs (POI.IARD \& LOGAN, 1977), que l'intensité d'initiation est très sensible à deux facteurs du milieu, température et stress hydrique. La sélection pour la tolérance à ce stress pourrait donc être une sélection indirecte afficace, en particulier pour la recherche de critères prédicteurs juvéniles de la croissance. Cette constatation à elle seule pourrait expliquer le manque de corrélation juvénile adulte dans l'hypothèse où il $y$ aurait une interaction génotype $x$ milieu. Il serait donc intéressant en sćlection de tester dès le stade juvénile le matériel végétal en condition de stress. Dans 
ce cadre, les caractéristiques géométriques du méristème apical (dimensions du dôme et géométrie de la phyllotaxie) peuvent s’avérer être des marqueurs efficaces de l'intensité de fonctionnement du méristème.

Reçu le 12 octobre 1984. Accepté le 18 février 1985.

\begin{abstract}
Summary
Subdivision of shoot growth of maritime pine (Pinus pinaster Ait.)

Geographic variation of morphogenetical and phenological commonents
\end{abstract}

The annual height increment in maritime pine is subdivided in four morphogenetical or phenological steps from the shoot to the apical meristem. The objective is the search of components that contribute for the most part to the resultant character. The method is then applied to nine provenances originating from the whole natural range of the species. Primary shoot length corresponding to the first morphogenetical cycle is a better predictor of annual height increment than secondary shoot length. Number of stem units is a better predictor of primary shoot length than mean stem unit length. Important differences between provenances exist for the duration and rate of functioning of the meristems responsible for the initiation and elongation of stem units. Transverse relative growth rates of the primordia and the bare apical dome are well correlated with the total number of stem units initiated during the season.

\title{
Références bibliographiques
}

Bailey D.B., Ferte P.P., 1982. Shoot elongation in Pinus rigida $\times$ taeda hybrids. Silvae Genet., 31, 209-212.

CANnEl, M.G.R., 1976. Shool apical growth and cataphyll initiation rates in provenances of Pinus contorta in Scotland. C'an. J. For. Res., 6, 539-556.

Canniti. M.G.R., Whllite S.C., 1975. Rates and times at which needles are initiated in buds on differing provenances of Pints contorta and Picea abies in Scotland. Catr. $J$. For. Res., 5, 367-380.

Cannill. M.G.R., Thomeson S., Lints R., 1976. An analysis of inherent differences in shoot growth within some north temperate conifers. In : Tres physiology and yield improrement. Eds. M.G.R. Cannell and F.T. Last, Academic Press, 173-205.

Cannell M.G.R., 1978. Components in conifer shoot growth. In : Proceedings of the 5th north american forest biology workshop. Edited by C.A. Hollis and A.E. Squillace. University of Florida, Gainesville, 313-318.

Canneli. M.G.R., Cahalan C.H., 1979. Shoot apical meristems of Picea sitchensis seedlings accelerate in growth following bud set. Ann. Bot., 44, 209-214.

Causion D.R., Venus J.C., 1981. The biometry of plant growth. Edward Arnold, 307 p.

Debazac E.F. 1963. Morphologic et sexualité chez les pins. Rev. For. Fr., 14, 293-303.

Distrimau D.X., Joll. Y H., 'TahlRI T., 1974. Contribution à la connaissance des provenances de Pinus pinaster. Ant. Rech. For. Maroc., 16, 101-153.

Gregory R.A., Rombi:kitik J.A., 1972. The shoot apical ontogeny of the Picea abies seedling. II Growth rates. Am. J. Bot.. 59, 598-606.

Guyon J.P., 1980. Variabilité géographique ef écophysiologique da pin maritime. Mémoire de $3^{\prime \prime}$ année. E.N.I.T.E.F., Nogent-sur-Vernisson.

Guyon J.P., Krimer A., 1982. Stabilité phénotypique de la croissance en hautcur et cinćtique journalière de la pression de sève et de la transpiration chez le pin maritime (Pinus pinaster Ait.). Com. J. For. Res., 12, 936-946.

Jean R.V., 1978. Phytomathématique. Les Presses de l'université du Québec, 274 p. 
Lascoux D.M., 1984. Décomposition de la croissance en hauteur du pin maritime. (Pinus pinaster Ait.). Aspects morphogénétiques et génétiques. Mémoire de 3" année, E.N.I.T.E.F., Nogent-sur-Vernisson.

LEBRETON J.D., Muliter C., 1982. Modèles dynamiques déterministe's én biologie. Masson, $208 \mathrm{p}$.

Kozlowski T.T., 1964. Shoot growth in woody plants. Bot. Rev., 30, 335-392.

Kremer A., 1982. Déterminisme génétique de la croissance en hauteur du pin maritime (Pinus pinaster Ait.). 1. Rôle dù polycyclisme. Ann. Sci. For., 38, 199-222.

Kremer A., 1984 a. Distribution of relative growth rates and variation of cytohistological zonation in apical meristems of seedlings of two contrasting open-pollinated jack pine (Pinus hanksiana L.) families. Can. J. For. Res., 14, $297-311$.

KREMER A., 1984 b. Component analysis of height growth, compensation between components and seasonal stability of shoot elongation in maritime pine (Pinus pinaster Ait.). Proceedings of the I.U.F.R.O. Conference on «Crop physiology of forest trees ». Helsinki (sous presse).

Kremik A., Roussi: (i., 1982. Composantes de la croissance en hauteur clie\% le pin maritime. Ann. Sci. For., 39, 77-98.

Krimer A., LARSON P.R., 1983. Genetic control of height growth components in jack pine seedlings. For. Sci., 29, 451-464.

OWEnS J.N., Mot.dir M., 1975. Development of long shoot terminal buds of Pintes contorta ssp. contorta. In : Proceedings of management of lodgepole pine ecossistem sympresiam. Eds D.M. Baumgartner, Washington State University, Pullmann, 86-104.

Pollard D.F.W., 1973. Provenance variation in phenology of needle initiation in white spruce. Can. I. For. Res, 3, 589-593.

Pollard D.F.W., Logan K.T., 1977. The effect of light intensity photoperiod, soil moisture potential and temperature on bud morphogenesis in Picea species. Car. J. For. Res., 7, $41.5-421$.

Riciards F.J., 1959. A flexible growth function for empirical use. J. E.xp. Bot., 10, 290)-300.

THOMPSON S., 1974. Shoot clongation and dry matter production in tho contrasted provenances of Pinus contorta. Ph. D. Thesis, University of Aberdeen.

Thornley J.H.M., 1975. Phyllotaxis : II. A description in intersecting logarithmic spirals. Amr. Bot., 39, 509-534.

Van den Bergi D.A., Lanni:r R.M., 1971. Bud development in Lodgepole pine. For. Sci., 17, 479-486.

WAREING P.F., 1970. Growth and it's coordination in trees. In : Physiology of rece crops, Academic press, 1-21.

WII.IAMS R.F., 1975. The shoot apex and leaf growth. Cambridge University press, $256 \mathrm{p}$. 